Trinity University

Digital Commons @ Trinity

Psychology Faculty Research

Psychology Department

$11-2011$

\title{
Recollection Is Impaired by the Modification of Interpretation Bias
}

\author{
Paula T. Hertel \\ Trinity University, phertel@trinity.edu \\ Elaina Vasquez \\ Trinity University \\ Amanda Benbow \\ Trinity University \\ Megan Hughes \\ Trinity University
}

Follow this and additional works at: https://digitalcommons.trinity.edu/psych_faculty

Part of the Psychology Commons

Publication Details

Journal of Abnormal Psychology

\section{Repository Citation}

Hertel, P.T., Vasquez, E., Benbow, A., \& Hughes, M. (2011). Recollection is impaired by the modification of interpretation bias. Journal of Abnormal Psychology, 120(4), 902-910. doi: 10.1037/a0023974

This Article is brought to you for free and open access by the Psychology Department at Digital Commons @ Trinity. It has been accepted for inclusion in Psychology Faculty Research by an authorized administrator of Digital Commons@ Trinity. For more information, please contact jcostanz@trinity.edu. 


\title{
Recollection Is Impaired by the Modification of Interpretation Bias
}

\author{
Paula T. Hertel, Elaina Vasquez, Amanda Benbow, and Megan Hughes \\ Trinity University
}

\begin{abstract}
The interpretation paradigm of cognitive-bias modification (CBM-I) was modified with instructions used in process-dissociation procedures for the purpose of investigating processes contributing to performance on the transfer task. In Experiment 1, nonanxious students were trained to interpret ambiguous situations in either a negative or benign way (or they read nonambiguous scenarios). They were then asked to respond to new ambiguous situations, in the same way as contextually similar analogues during training, or to respond differently. Benign training proactively impaired memory for negative outcomes. This effect was replicated by anxious students in Experiment 2 and discussed with respect to the assumptions underlying process-dissociation procedures and directions for future research.
\end{abstract}

Keywords: anxiety, cognitive bias modification, interpretation, memory

Since the beginning of the century, clinical researchers have been experimentally simulating interpretive biases that characterize cognition in mood-disordered states. Negative interpretive biases are revealed when events with ambiguous meaning - a frown, a laugh, a noise, a remark - are understood as emotionally negative, and anxious people, in particular, have demonstrated such bias (see Blanchette \& Richards, 2010; Mathews \& MacLeod, 2005). Efforts to simulate interpretation bias in nondisordered participants have been motivated by the desire to understand whether negative ways of perceiving and thinking play a causal role in the development of mood disorders instead of merely being produced by them.

The paradigm used to simulate interpretation biases is called the interpretation version of cognitive bias modification (CBM-I); it begins with many trials designed to lead participants to respond in a consistently benign or negative way, proceeds with an ambiguous transfer task to allow the free expression of the trained bias, and sometimes concludes with a measure of participants' emotional state. Among the different versions of the paradigm is a frequently used procedure invented by Mathews and Mackintosh (2000) in which a large number of short verbal scenarios used during training (e.g., 100) are ambiguous until their very last word; participants are led to end each scenario with a benign or negative resolution, depending on their experimental condition, by completing a word fragment. The transfer task consists of new scenarios that remain ambiguous; typically, participants reveal their interpretation by rating the similarity of new valenced sentences to the meaning of each transfer scenario. Reports of CBM-I experiments often imply that the training phase at least temporarily establishes

This article was published Online First June 13, 2011.

Paula T. Hertel, Elaina Vasquez, Amanda Benbow, and Megan Hughes, Department of Psychology, Trinity University.

The authors thank Ahra Cho for valuable assistance in preparation of materials and data collection.

Correspondence concerning this article should be addressed to Paula T. Hertel, Department of Psychology, Trinity University, 1 Trinity Place, San Antonio, TX 78212. E-mail: phertel@trinity.edu a new habit-one much like the habits that characterize anxietydisordered cognition. The motivation for the experiments we report is the realization that another consequence of training is also possible. Performance on a transfer task can be guided by recollection of prior training as well as by habit. More generally, we intended these experiments as a first step in investigating the procedural bases of transfer and, thereby, as an important next step in understanding the contribution of interpretation biases to emotional disorders. Knowing about the underlying processes that give rise to CBM-I effects will permit a fuller integration of these findings with cognitive theories of emotional disorders (see MacLeod, Koster, \& Fox, 2009).

CBM-I experiments find their place in a long tradition of experiments on transfer of learning (see Ellis, 1965). Transfer experiments, regardless of their ilk, reveal effects of training on performance in subsequent similar tasks with different content. Similarity thus provides the basis for habitual or recollective influences of training. When faced with an ambiguous burst of laughter during a speech, for example, a socially anxious person might remember having had a similar experience in the past during which she felt humiliated, or she might habitually interpret the laughter as scorn, with no thought of similar prior events. Quite possibly, however, some combination of recollection and habit guides her interpretation. As a first approach to examining the bases of transfer in CBM-I tasks, our experiments used Jacoby's process-dissociation procedure (PDP; Jacoby, 1991).

Process-dissociation procedures were developed partly in response to questions about the "process purity" of performance on tests of implicit memory (Jacoby, 1991). Because participants are not instructed to think back to prior experience as they perform on an implicit memory test, automatic influences of prior experience had been assumed to guide performance, much as they are assumed to underlay CBM-I effects on transfer performance, although, in both cases, recollection could be spontaneously instigated by some noninstructed means. A typical process-dissociation paradigm utilizes two types of instructions on test trials: one in which participants are asked to respond with an item encountered previously and one in which they are asked to try to recall a 
previous instance and respond differently. If they fail to respond differently in the latter case, against instructions, then the influence of prior experience might lie outside awareness, as habits often do. PDP instructions culminate in estimates of automatic and controlled components of memory when the assumption about their stochastic independence can be met. We provide these estimates; however, our main interest is in the analogy between task instructions and ways in which people use prior experience to interpret current ambiguity. To be consistent with CBM-I phases of training and transfer, we modified the PDP paradigm by asking participants to respond in the same way as they had previously responded to a similar scenario (by choosing between negative and benign completions of ambiguous test scenarios) or to try to remember a similar scenario and respond differently. These instructions serve as experimental analogues to what anxious people might be asked to do in treatment: to respond to new ambiguous situations in a manner that is similar to the way they have practiced in therapeutic contexts, or to respond differently from the way they typically respond.

We report two experiments performed with CBM-I procedures modified by PDP instructions at test. The training phase was designed like prior CBM training phases (see Mackintosh, Mathews, Yiend, Ridgeway, \& Cook, 2006; Mathews \& Mackintosh, 2000). Ambiguous scenarios were each resolved by the participants' completion of a fragment of the final word, followed by the answer to a yes/no question about the scenario (to require comprehension). For example,

You are going to the nearest store to get one or two items. As you pick them out two men come in and walk to the checkout. You walk up behind them and hear one of them is demanding: m-n-y/s-rv-ce (money/service).

Q: Are the two men trying to rob the store?

The training phase was followed by a critical phase that contained additional training scenarios devised to serve as targets for the test. Pairs of scenarios were created for the critical and test phases, with one member to be allocated to the critical phase and the other to the test. The members of each pair shared a similar context such that it was possible, when presented with the test member of the pair, to think back to a similar pair in the critical phase. Table 1 contains examples. During the critical phase, the resolution of ambiguity was forced by fragment completion; during the test phase, participants were instructed to respond in the same or a different way, compared to how they had responded to a similar item encountered during the most recent task.

In the first experiment, for ethical reasons, we recruited students who scored low on a self-report test of trait anxiety and randomly assigned them to training conditions that encouraged a negative or benign interpretation bias or to a control condition in which they responded to nonambiguous scenarios with little potential for negative interpretation. Because we were initially unsure about the number of scenarios we could use without confusing participants, the test phase contained scenarios corresponding only to negative resolutions, although the critical phase contained both negative resolutions and benign fillers to disguise the rule. For the second experiment, we recruited students who scored high in trait anxiety and assigned them to either benign training or the control condition and tested with scenarios corresponding to both negative and benign resolutions from the critical phase.

At the outset of these experiments, we entertained three possible outcomes. The common assumption in the CBM literature is that the trained bias is automatic (see MacLeod et al., 2009; Mathews, 2010), in which case participants should have a difficult time responding differently from how they responded during training. It seemed possible, however, that prior experience during training could affect recollection of critical scenarios through elaborative focus on outcomes consistent with training, in which case participants should more accurately respond in the same way as they had during training. Indeed, there is some evidence that awareness of the nature of interpretation training mediates transfer (Salemink, van den Hout, \& Kindt, 2007). In the third possible outcome, training might cause proactive interference in memory for training-incongruent resolutions. A possible real-world analogue exists in the case of nonanxious individuals protected from remembering specific threatening outcomes by their prior experience with benign resolutions. The results of Experiment 1 were consistent with this last possibility.

\section{Experiment 1}

\section{Method}

Overview and design. All participants were randomly assigned to negative or benign training (or to a control condition), in

Table 1

Example Scenarios From the Critical Phase and Corresponding Test Scenarios

\begin{tabular}{|c|c|}
\hline Critical phase & Test phase \\
\hline $\begin{array}{l}\text { You are flying to Florida with your family for a } \\
\text { holiday in the sun. You notice a man sitting } \\
\text { alone in the row behind you, opening a } \\
\text { package. As the paper is removed you see } \\
\text { something that looks like a: bo-klbo-b. } \\
\text { [book lbomb] }\end{array}$ & $\begin{array}{l}\text { You are going to Spain for a week and } \\
\text { feel excited as the plane takes off. In } \\
\text { the seat beside you is a burly man and } \\
\text { you can't help noticing a bulge under } \\
\text { his jacket. As the stewardess comes by, } \\
\text { he pulls out a: grenade`newspaper }\end{array}$ \\
\hline $\begin{array}{l}\text { You have agreed to baby-sit while your neighbor } \\
\text { goes out for the evening. You have put their } \\
\text { daughter to bed but she takes some time to } \\
\text { settle down. An hour later you go in to check } \\
\text { on her and find she is no longer: cr-ing } \\
\text { c-nsc-ous [cryinglconscious] }\end{array}$ & $\begin{array}{l}\text { A neighbor asks you to look after her little } \\
\text { girl while she visits a friend in the } \\
\text { hospital. The five year old cries when } \\
\text { her mother leaves but then seems happy } \\
\text { to play alone in your garden. After ten } \\
\text { minutes you go out and she is: gonelfine }\end{array}$ \\
\hline
\end{tabular}


which they read potentially negative (physically threatening) scenarios and completed a fragment of the last word in each one, thereby resolving the meaning of the scenario as negative or benign. Following a pause for brief instructions, they continued responding to similar scenarios in the critical phase. This phase contained the analogs to test scenarios; we separated them from training in order to provide some contextual basis for remembering during the test. The critical phase consisted of 12 critical scenarios resolved as negative by all participants, regardless of training, and nine benign fillers to disguise the rule. (Again, we did not vary the valence of critical and test scenarios because we were initially unsure about the number of scenarios we could use without confusing participants.) The test phase followed immediately and consisted of 12 ambiguous scenarios, each analogous to a critical scenario and followed by two options for endings. Participants were instructed to remember back to the previous phase and respond in a similar or different manner (for six scenarios each). Mood scales were completed before training and after the test.

Participants. Forty-eight students at Trinity University ( 24 of each gender) participated in the experiment for extra credit in their introductory psychology course. Students completed the trait form of the State-Trait Anxiety Inventory (STAI; Spielberger, Gorsuch, Lushene, Vagg, \& Jacobs, 1983) during class and were subsequently selected to participate if their trait scores fell at the median of 39 or lower (ranging from 23 to 39). They were randomly assigned to training conditions and to one of four counterbalancing conditions for the test phase, given the constraint of equal cell sizes according to gender. Assignment toward the end of the recruitment period considered the values of the trait scores in an attempt to balance the three groups $\left(M_{\text {negative }}=30.8, S D=4.05 ; M_{\text {benign }}=\right.$ $\left.30.9, S D=4.20 ; M_{\text {control }}=31.6, S D=3.12\right)$. The data from six participants were replaced due to their failures to understand the instruction for the test $(n=3)$, poor compliance during training $(n=2)$, or experimenter error $(n=1)$.

Materials. Scenarios for the training phase consisted of 60 training scenarios, 10 irrelevant fillers, and 20 probe scenarios (10 benign and 10 negative). Time to complete the fragments on probe trials was used to assess the effects of training independently from transfer. The critical phase employed 12 scenarios resolved negatively and 9 fillers with benign endings; 12 test scenarios each corresponded in context to a critical negative scenario.

Training phase. The 60 ambiguous training scenarios in the negative and benign conditions were based on those used by Mackintosh et al. (2006). They described situations containing the potential for threat to one's safety or health. On average, they occupied three lines of text, with the last word presented in fragmented form. Each fragmented word had only one possible solution, and it disambiguated the text in either a negative or benign manner-for example, You are getting ready to go out and look at yourself in the mirror. You notice a brown mark on your face that you do not remember seeing before. It is very small and you realize it may actually be: m-lign-nt/at-rac-ive (malignant) attractive). Each scenario was followed by a simple comprehension question, the answer to which validated the correct interpretation of the scenario (e.g., Do you think that the brown mark could be dangerous?).

Of the 20 ambiguous probe scenarios, 10 led to fragments that resolved in the negative direction and 10 in the benign direction, regardless of training condition; they were identical in the two conditions. Used to help conceal the training manipulation, nonambiguous fillers were low in emotional content. All materials were presented in 10 fixed blocks, each containing six training scenarios, two probe scenarios, and one filler. The order within blocks was randomized anew for each participant.

To expose participants in the control condition to the process of reading scenarios and responding to the fragments, we presented them with 40 nonambiguous scenarios in a random order-for example, You begin reading a book that you recently found around your house. One afternoon you are reading it while sitting in your recliner. You start to feel hungry so you put the book: $d$-wn.

Critical phase. Twelve critical scenarios served as the to-beremembered target events during the test phase. Their ambiguity with respect to physical threat was always resolved through completion of a fragment for a negative word. The nine filler scenarios, included to obscure the fact of negative resolutions for critical scenarios, were also ambiguous but resolved in the benign direction. Questions were not used in this phase out of concern that they might incur ceiling levels of recollection. These scenarios were assigned to one of three blocks, each containing four negative and three benign scenarios. Block order was fixed and scenario order randomized within blocks.

Test phase. Each of the 12 ambiguous test scenarios was similar in content to one of the critical scenarios (examples in Table 1). Each was followed by a screen displaying two possible completions-one negative and one benign-arranged horizontally. The negative alternatives never replicated the fragmented words from the corresponding critical items, although they invoked the same resolution of ambiguity. Scenarios were presented in three randomized blocks of four each; block membership was held constant across the critical and test phases. The four scenarios in each test block varied according to whether the negative word was presented on the left or the right and whether the scenario was presented in green font for same or red font for different instructions (one of each combination within each block). These values were rotated across materials, producing the four counterbalancing conditions.

Mood forms. To collect mood ratings, we used visual analogue scales (VAS: depressed to happy, tense to relaxed, pessimistic to optimistic, distressed to not distressed). Each scale was presented as a horizontal line labeled at each end; participants drew a vertical line to indicate their current mood. The scales were each scored from 1-100 (1 = depressed, tense, pessimistic, distressed $; 100=$ happy, relaxed, optimistic, not distressed).

Procedure. Following informed consent, the participants rated their current mood on the VAS. Each phase of the CBM session began with instructions and two practice examples. In the training phase, we instructed participants to imagine themselves in the situation described in each scenario and to anticipate its final word and press the space bar to reveal the fragment. They typed the word and clicked an arrow labeled "next" to reveal the question, to which they responded by pressing the "y" key (for yes) or the " $n$ " key (for no).

After completing 90 training scenarios, the participants turned away from the monitor while the experimenter initiated the critical phase. Participants were told that a much shorter set of scenarios would be presented next, this time without questions, and they should read carefully because this was an important part of the experiment. Test scenarios immediately followed a similar instruc- 
tional interlude after the critical phase. We told participants that each test scenario was similar in content to one of the scenarios from the previous phase and the way they should respond to these scenarios depended on both the way they had responded to the similar scenario and the color of the font in which the text was presented. If it was green, they should respond in the same way as previously; if red, they should respond differently. After reading each scenario, participants pressed the spacebar to reveal the two alternatives for completion (negative and benign). To choose the word on the left, participants pressed the "v" key and to choose the word on the right they pressed the "b" key, each marked with red dots. Following the test phase, participants rated their current mood again.

\section{Results and Discussion}

First, we present the results in terms of the proportion of negative choices on both same and different trials. Then we use these proportions to estimate automatic and recollective components of transfer performance. The significant level was set at .05 for all analyses in this report.

Proportion of negative resolutions. Proportions of negative resolutions chosen at test were submitted to a mixed-design analysis of variance (ANOVA), with a between-subjects factor for training (negative, benign, control) and a within-subjects factor for instruction at test (same, different). Means are presented in Table 2. Revealing that the instructions were effective in the context of CBM-I, the main effect of instruction was significant and large, $F(1,45)=84.57, M S E=.056, p<.001, \eta_{\mathrm{p}}^{2}=.65$. More important, the interaction was also significant, $F(2,45)=10.53$, $p<.001, \eta_{\mathrm{p}}^{2}=.32$, and followed by tests of simple main effects within each instruction.

Same trials. The three training groups differed when participants were asked to respond in the same way as they had to a previous, critical scenario, $F(2,45)=5.79, M S E=.048, p=.006$, $\eta_{\mathrm{p}}^{2}=.20$. Negatively trained participants correctly chose more negative outcomes than did controls, but not significantly so, $p=$ .23; those with benign interpretation training chose significantly fewer than controls, $p=.037,95 \%$ CI $[-.32,-.01]$. Thus, benign training established proactive interference in memory for negative outcomes.

Different trials. The three training groups also differed when participants were asked to remember a corresponding scenario and respond differently, $F(2,45)=6.31, M S E=.050, p=.004, \eta_{\mathrm{p}}^{2}=$ .22. Again, there was a nonsignificant difference between negatively trained and control conditions, $p=.360$, but compared to controls, those with benign training were less successful at avoiding the previous resolution of ambiguity, $p=.016,95 \% \mathrm{CI}$ $[-.357,-.039]$. The relative failure to respond differently can

Table 2

Experiment 1: Mean Proportion of Negative Resolutions of Ambiguity During Test (Standard Deviation)

\begin{tabular}{lcc}
\hline Training condition & Same instruction & Different instruction \\
\hline Negative & $.79(.206)$ & $.11(.208)$ \\
Benign & $.53(.229)$ & $.39(.296)$ \\
Control & $.70(.221)$ & $.19(.134)$ \\
\hline
\end{tabular}

result from a strong habit to endorse negative outcomes, but benign training or low trait anxiety does not establish such a habit. We therefore suggest that the source of this difference again lies in memory for the prior outcome.

Estimates of recollection and automaticity. The results just reported should not be interpreted as reflecting unitary cognitive processes. In addressing issues of the process purity of performance on implicit tests of memory, Jacoby (1991) assumed that recollective and automatic uses of prior experience make independent contributions to performance on any test. If the independence assumption can be made in Experiment 1, on same trials, participants completed the test scenarios by choosing negative outcomes because they recollect the previous outcome $(\mathrm{R})$ or because, in the absence of such recollection, habit guided the choice $[(1-\mathrm{R}) \mathrm{H}]$; the probability of correctly responding on same trials is therefore $\mathrm{R}+(1-\mathrm{R}) \mathrm{H}$. On different trials, participants responded erroneously with negative outcomes when, in the absence of recollection, habit guided the choice $[(1-\mathrm{R}) \mathrm{H}]$. To obtain estimates of $\mathrm{R}$, the proportion of different trials on which negative outcomes are erroneously chosen are subtracted from the proportion of same trials on which negative outcomes are correctly chosen. Estimates of $\mathrm{H}$ are obtained by substitution. Estimates of $\mathrm{R}$ and $\mathrm{H}$ can then be examined for effects of cognitive bias modification.

We present the analysis of these estimates even though we cannot verify the assumption that recollection and habit operate similarly in response to the two instructions. This assumption is typically validated by examining performance on test items that are new to the participants (and counterbalanced with "old" test items across participants). In the CBM-I paradigm, new test trials are difficult and perhaps impossible to invent, due to the great degree of similarity among training items and the fact that old items are actually never used at test because the test is a transfer test containing all new items. "New" test scenarios would have to be dissimilar to all training and critical items yet contain the possibility for a negative resolution of ambiguity. Lacking such items, we cannot evaluate the assumptions necessary for process estimates, and so we present the analyses merely as possibilities to be considered.

Estimates of controlled recollection. To paraphrase Jacoby (1991) for our purposes, controlled, recollective use of prior experience can be conceived in terms of both successfully responding in the same way as previously and successfully avoiding the prior response when it is undesirable. Estimates were obtained by subtracting the proportion of negative choices on different trials from the proportion of negative choices on same trials. The analysis of these estimates corresponds exactly to the test of the interaction of training condition with instruction, reported previously. Mean estimates are shown in Figure 1. Compared to the control condition, negative training did not improve recollection, but benign training impaired it, $p=.004,95 \%$ CI $[-.61,-.13]$.

Estimates of habit. Habit estimates were obtained by dividing the proportion of negative choices under instructions to respond differently by $(1-\mathrm{R})$. This operation produced indeterminate results for four participants in the negative training condition because recollection was at ceiling; therefore, the mean estimate in this condition is potentially misleading $(M=.27, S D=.267)$. Nevertheless, the differences among the three training conditions were nonsignificant, $F(2,41)=1.44, M S E=.086, p=.248$, $\eta_{\mathrm{p}}^{2}=.07\left(M_{\text {control }}=.44, S D=.330 ; M_{\text {benign }}=.44, S D=.271\right)$. 


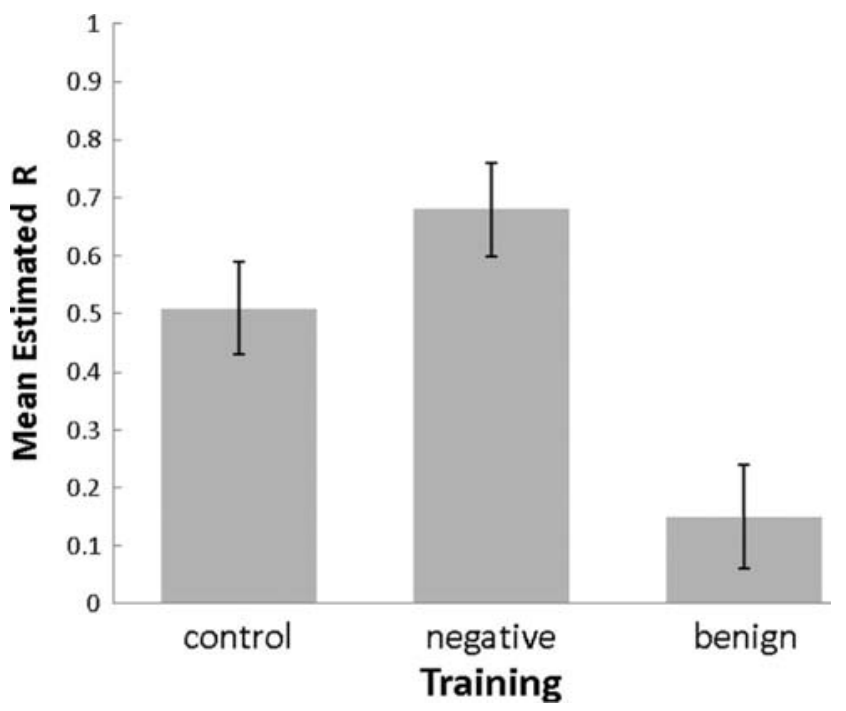

Figure 1. Mean estimates of recollection in each training condition in Experiment 1. Error bars represent one standard error.

No evidence for a trained habit was found; however, this outcome should be considered quite cautiously, because assumptions could not be validated in this instantiation of PDP.

Measures during training. Time to complete the fragments on probe trials served as an independent measure of transfer. Latencies in $\mathrm{ms}$ were measured from the key press that revealed the fragment to the striking of the first key of the response. Mean latencies were submitted to a mixed-design ANOVA, with the data from four participants missing due to experimental error (three in the negative and one in the benign condition). The design included a between-subjects factor for training and within-subjects factors for valence and half (first vs. second half of the 10 negative and 10 benign probe trials). The analysis revealed a marginally significant interaction of training and valence, $F(1,26)=4.09, M S E=$ 239521, $p=.054, \eta_{\mathrm{p}}^{2}=.14$. Negatively trained participants responded similarly to negative and benign probes $\left(M_{\text {negative }}=1632\right.$, $M_{\text {benign }}=1622$ ), but those in the benign condition responded more slowly to negative probes $\left(M_{\text {negative }}=1993, M_{\text {benign }}=1608\right) .{ }^{1}$ Finally, along with the main effect of valence and the main effect of half, the interaction of valence with half was significant, $F(1,26)=$ $9.43, M S E=181627, p=.005, \eta_{\mathrm{p}}^{2}=.27$. Across training conditions, these nonanxious participants responded more slowly to negative than benign resolutions in the first half $\left(M_{\text {negative }}=2267, M_{\text {benign }}=\right.$ 1822), but the difference disappeared during the second half $\left(M_{\text {negative }}=1357, M_{\text {benign }}=1408\right)$. Taken together, these results indicate that $\mathrm{CBM}$ was somewhat successful.

The number of correctly answered questions on probe trials served as a check for compliance in reading the scenarios. As might be expected from nonanxious participants, a larger proportion of questions were answered correctly on benign probe trials during training than on negative probe trials, $F(1,30)=5.83$, $M S E=.015, p=.022, \eta_{\mathrm{p}}^{2}=.16\left(M_{\text {benign }}=.90, M_{\text {negative }}=.83\right)$. Yet, this difference did not depend on training, and there was no overall difference according to training, $p>.58$.

Mood change. Separate mixed-design ANOVAs were conducted for each of the mood scales, with time of measurement as the within-subjects factor and training condition as the between-subjects factor. The pessimistic/optimistic scale was the only one to produce significant differences; all other $p$ values $>.383$. Participants became more pessimistic as a result of experimental events, $F(1,45)=4.21$, $M S E=111.21, p=.046, \eta_{\mathrm{p}}^{2}=.09\left(M_{\text {Time } 1}=75, M_{\text {Time } 2}=70\right) . \mathrm{In}$ addition, the analysis revealed a nonsignificant trend for an interaction of time with training condition, $F(2,45)=2.73, p=.076, \eta_{\mathrm{p}}^{2}=.11$. The increase in pessimism found in the other conditions was absent in the benign condition, but participants in this condition began the session in a more pessimistic frame of mind $\left(M_{\text {Time } 1}=66, M_{\text {Time } 2}=\right.$ 69) compared to those in the negative condition $\left(M_{\text {Time }{ }_{1}}=75\right.$, $\left.M_{\text {Time 2 }}=67\right)$ and control condition $\left(M_{\text {Time 1 }}=82, M_{\text {Time } 2}=74\right)$. In short, we found little evidence of change in mood state as a function of our procedures.

\section{Experiment 2}

The results of Experiment 1 encouraged us to perform a second experiment in order to replicate the proactive interference effect with anxious individuals, for whom benign training has been successful in previous experiments (see Mathews, 2010). In addition, we had planned from the outset to include benign test trials in a second experiment after exploring the levels of performance obtained with 12 test trials in Experiment 1. The issue concerned whether participants could remember specific content after having read so many scenarios during training. In that regard, responsiveness to instructions as well as end-of-session interviews indicated that participants subjectively experienced recall of the relevant critical scenarios. Therefore, we added new scenarios to the critical and test phases in Experiment 2. Finally, the method of instructing participants about how to respond on the test was changed to a one-word verbal display (same or different) presented just before the alternatives.

\section{Method}

Participants and design. Forty introductory psychology and introductory neuroscience students at Trinity University (20 of each gender) participated in the experiment for credit in their courses. Students completed the trait version of the STAI and were selected to participate if they produced scores above the median of 39. (Actual scores ranged from 43 to $68 ; M_{\text {benign }}=50.6, S D=$ $\left.5.71 ; M_{\text {control }}=50.1, S D=6.0\right)$. With the constraint of equal cell sizes, participants were randomly assigned to receive either benign or control training and to one of four counterbalancing conditions for rotating instructions and position across items on the test.

Materials and procedure. As in Experiment 1, participants in the benign condition read 90 scenarios in the training phase (60 training, 10 fillers, and 20 probes). Those in the control condition read a total of 70 scenarios organized in 10 blocks of seven (five nonambiguous scenarios plus one negative and one positive probe scenario). Half of the 24 scenarios in the critical phase ended with negative resolutions and half with benign. Seven new scenarios were added to those used in Experiment 1, four were deleted, and others were slightly modified. The resolution of ambiguity in each

\footnotetext{
${ }^{1}$ The simple interaction of training condition with valence was significant in the second half, $F(1,26)=5.38, M S E=83763, p=.029, \eta_{\mathrm{p}}^{2}=$ .17 , but the pattern did not significantly differ from the first half.
} 
scenario was reversed from Experiment 1. Scenarios were presented in three randomized blocks of eight scenarios each (four negative and four benign). The test phase was composed of 24 scenarios corresponding to those presented in the critical phase and was organized according to the same randomized-block design, preserving the same block position as the paired scenarios from the critical phase. Within blocks, the four of each resolution type represented the combinations of position (negative resolution on left or right) and instruction (same or different). Test scenarios were presented in black letters. A new screen with the centered instruction SAME or DIFFERENT appeared for $1500 \mathrm{~ms}$ after the participant had read the scenario and pressed the spacebar; this instruction preceded the presentation of the two alternative resolutions of ambiguity.

After the second mood scale (following the test), we showed short clips from the film What Lies Beneath (Zemeckis, Starkey, \& Rapke, 2000). The scenes involved an apprehensive woman watching her front door open by itself, her bathtub mysteriously fill with water, and writing appear on her foggy bathroom mirror. A third set of mood scales followed the clips. This was our attempt to utilize a stressor that might be capable of revealing differences in emotional reactions as a function of training (see Wilson, MacLeod, Mathews, \& Rutherford, 2006). In all other respects, the materials and procedures were the same as those used in Experiment 1 .

\section{Results and Discussion}

Proportion of negative and benign resolutions. Proportions of test choices that conformed to the resolution forced during the critical phase were submitted to a mixed-design ANOVA, with a between-subjects factor for training (benign, control) and withinsubjects factors for instruction at test (same, different) and valence of the critical resolution (negative, benign). Means are presented in Table 3. The three-way interaction was significant, $F(1,38)=$ 9.87, MSE $=.030, p=.003, \eta_{\mathrm{p}}^{2}=.21$. (The main effect of instruction was also significant, $p<.001$.) Follow-up tests of simple interactions within each instruction are described next.

Same trials. The proportion of test choices that conformed to the resolution forced during the critical phase depended on the interaction of training and resolution valence, $F(1,38)=6.64$, $M S E=.035, p=.014, \eta_{\mathrm{p}}^{2}=.15$. Replicating the effect in Experiment 1, benign training decreased the proportion of correct negative resolutions, $t(38)=2.26, S E=.063, p=.029,95 \% \mathrm{CI}$ $[-.27,-.02]$. A nonsignificant benefit of benign training was found in the correct production of benign resolutions, $p=.194$;

Table 3

Experiment 2: Mean Proportion of Negative and Benign Resolutions of Ambiguity During Test (Standard Deviation)

\begin{tabular}{lcc}
\hline Resolution valence & Same instruction & Different instruction \\
\hline Negative resolutions & & \\
Benign training & $.61(.197)$ & $.33(.226)$ \\
Control & $.75(.199)$ & $.23(.198)$ \\
Benign resolutions & & \\
Benign training & $.78(.109)$ & $.30(.192)$ \\
Control & $.71(.229)$ & $.33(.202)$ \\
\hline
\end{tabular}

however, benign training produced more correct benign than negative resolutions, $t(19)=3.94, S E=.044, p=.001,95 \%$ CI $[.08$, .27].

Different trials. The simple interaction of training and valence was not significant, $p=.118$. An a priori test of the training difference in avoiding negative items (found in Experiment 1) was also nonsignificant, $p=.180$, although the difference was in the direction of replication. The lack of replication might reflect changes in procedure or levels of anxiety. More important to our conclusions, however, is the fact that in neither experiment did we find a pattern of avoidance on different trials that would suggest training-congruent habit.

Estimates of recollection and habit. Estimates were submitted separately to a mixed-design ANOVA with a between-subjects factor for training condition and a within-subjects factor for valence of the resolution in the critical phase. Estimates of habit did not differ significantly according to training for either type of resolution during the critical phase. As illustrated in Figure 2, estimates of recollection produced a significant interaction, $F(1$, $38)=9.96, M S E=.059, p=.003, \eta_{\mathrm{p}}^{2}=.21$. Compared to the control condition, benign training impaired recollection of negative resolutions, $t(38)=2.46, S E=0.095, p=.019,95 \% \mathrm{CI}$ $[-.43,-.04]$, but failed to improve recollection of benign outcomes, $p=.293$. Anxious participants in the control condition tended to recollect negative resolutions better than benign, although the difference was nonsignificant, $p=.113$. Those in the benign condition clearly recollected benign outcomes better than negative ones, $t(19)=3.00, S E=0.067, p=.007,95 \%$ CI $[.06$, .34]. Thus, the interference effect of benign training for negative outcomes found in Experiment 1 was replicated in a sample of anxious students. Anxious students in the control group showed an untrained tendency to recollect negative better than benign outcomes, and this difference was reversed by benign training.

Measures during training. Because critical trials with both outcomes were included in this experiment, we could assess training by examining performance in the critical phase, following the completion of the main training phase. This method was preferred, given the smaller number of probe trials during the main training phase and the reasoning that differences should be more likely found following a greater number of training trials. Latencies in $\mathrm{ms}$ to begin typing final words during the critical phase were submitted to a mixed-design ANOVA, with a between-subjects factor for training and a within-subjects factor for valence. The analysis revealed a significant interaction, $F(1,38)=4.45, M S E=64,862$, $p=.042, \eta_{\mathrm{p}}^{2}=.12$. Benign-trained participants responded more slowly on negative trials $\left(M_{\text {negative }}=1759, M_{\text {benign }}=1606\right), t(19)=$ 2.26, $S E=67.71, p=.036,95 \%$ CI $[10.9,294.4]$. Controls did not differentiate, $p>.350\left(M_{\text {negative }}=1610, M_{\text {benign }}=1697\right)$.

Change in mood and reactions to stressor. There were no significant training-related differences in mood ratings from the first to the second measurement. To investigate the possibility that CBM affected reactions to our film, we also analyzed changes from the second to the third measurement times (see Wilson et al., 2006). None of these analyses revealed significant interactions, $p>.50$. However, all participants reported more distress, pessimism, and tension after watching the clips. Changes varied from 5 points on the scale (pessimistic, $M_{\text {second }}=.56, M_{\text {third }}=.49, \mathrm{p}=$ $.006, \eta_{\mathrm{p}}^{2}=.18$ ) to 20 points (tense, $M_{\text {second }}=.52, M_{\text {third }}=.32$, $p<.001, \eta_{\mathrm{p}}^{2}=.49$ ). Either our choice of an appropriate stressor 


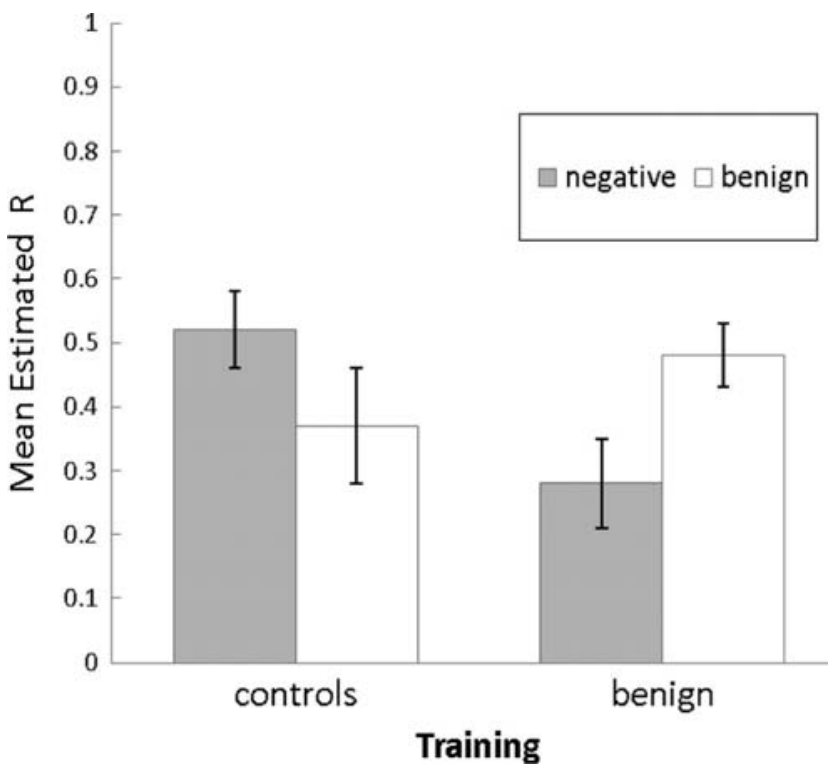

Figure 2. Mean estimates of recollection for scenarios that were resolved in negative or benign directions by participants who had been trained in the benign and control conditions in Experiment 2. Error bars represent one standard error.

was insufficiently ambiguous, sufficient power was lacking to detect a small effect, or CBM did not ameliorate emotional reactions.

\section{General Discussion}

A large number of CBM-I experiments have successfully simulated anxiety-related interpretation biases by guiding the resolution of many ambiguous situations toward threatening conclusions and observing negative interpretations of subsequent ambiguous situations. As precursors to therapeutic interventions, successful change in the opposite direction has also been found, even in anxiety-disordered samples (e.g., Beard \& Amir, 2008). The results from our experiments, designed to explore the bases of such transfer of training, suggest that one mechanism is impaired memory for negative resolutions brought about by benign training. When asked to respond similarly to how they had previously responded, nonanxious (Experiment 1) and anxious (Experiment 2) students in the benign training condition chose fewer negative resolutions when it was appropriate to do so. Unlike anxious controls, the anxious students with benign training also correctly chose more benign than negative resolutions, although they did not correctly choose more benign resolutions than did controls.

Performance following instruction to resolve in line with previous experience should not be assumed to reflect a unitary process: It can be affected by both habitual and controlled effects of that prior experience. For that reason, independence equations were used to separately estimate each component, even though the underlying assumptions for interpreting the equations could not be assured. Another way to view the estimates of recollection, however, is to realize that students who produced low estimates were less successful in both the intentional use and the intentional avoidance of the resolutions constrained at the end of training in
Experiment 1. In this sense, controlled recollection of negative outcomes was impaired by benign training. Pursuit of this finding, whether expressed in terms of performance measures or process estimates, might lead us to design effective cognitive vaccines for anxious people (see Holmes, Lang, \& Shah, 2009, for such a vaccine for depression).

Neither experiment produced evidence that habitual responding during transfer was affected by training. Although habit can logically contribute to correct responding on same trials, no evidence of training congruent performance was obtained on those trials. Moreover, when participants were instructed to respond differently, training-congruent errors were not observed. Instead, nonanxious students with benign training, compared to controls, unintentionally responded in a training-incongruent manner on different trials. (The same pattern, although nonsignificant, was also observed for anxious students.) Responding in the same manner when instructed to do the opposite can result both from a strong habit to respond that way (clearly not true of these participants) and from faulty memory for the outcome of the prior analogue. In the case of our participants with benign training, the latter source is consistent with their deficient performance when asked to respond in the same way.

One reasonable explanation for the lack of habit-related effects of CBM-I in these experiments is a lack of power. Many more training trials might be needed to show changes at the automatic level. The combination of our training and critical phases consisted of approximately the same number of training trials as other CBM experiments focused on scenario-based interpretation training (e.g., excluding probe trials, 72 negative trials in Experiment 1 and 72 benign trials in Experiment 2, compared to 64 by Mathews \& Mackintosh, 2000, and 80 by Mackintosh et al., 2006), although not as many as might be needed to reverse biases found in anxious populations. Thus, we advise against conclusions that habit is not trained in CBM-I, and we do so for yet another reason.

For the lack of habit-related effects in these experiments to be relevant to the interpretation of CBM-I outcomes in other experiments, we would need to be certain that the automatic component of transfer invoked by our PDP instructions is the same automatic component that operates without any instruction to think back to the prior phase during the transfer task, as is typical in CBM-I experiments. In other words, habit might operate differently under implicit instructions than under PDP instructions. (See Jacoby, 1991, for a discussion of these issues.) Nevertheless, automatic influences of prior experience are certainly what therapists wish to oppose when they encourage clients to think about their prior behavior and respond differently. Because the notion of opposing habit has ecological validity, the absence of a training congruent pattern on different trials is notable. Pursuit of this issue with a greater amount of training is an important direction for future CBM research.

In spite of the lack of habit-related effects in our experiments, however, the general implication of our main finding of proactive interference in memory for negative resolutions is oddly synchronous with what would be implied by a change in habit. If we cannot change habits for the better, we can at least impair the ability to remember negative events. A recent meta-analysis found substantial evidence for selective recall of negative material by anxious individuals (Mitte, 2008). Even the anxious controls in Experiment 2 tended to recollect negative outcomes at higher 
levels than benign outcomes. Another important direction for CBM research, therefore, is to determine whether recollection of negative events prolongs or exacerbates anxiety disorders. If so, impairing recollection of negative resolutions might indeed be a worthwhile venture.

Although the results from our performance measures can be interpreted in straightforward ways, our findings expressed in terms of estimates of controlled and automatic processes stand on less firm ground. One limitation concerns the legitimacy of the assumption that habit and recollection make independent contributions to the resolution of ambiguity. Another possible assumption about these processes in the context of CBM-I is akin to the generate/recognize model of performance on a variety of memory tasks (see Jacoby, 1996). This model would be appropriate if resolutions of the test scenarios come to mind automatically and subsequently are checked for "recognition" of whether they correspond to the appropriate critical scenarios, following an evaluation of the instructions to respond similarly or differently. According to this model, the proportion of resolutions correctly generated under same instructions is a process-pure estimate of habit (or the probability of generation). In that regard, what we found in Experiment 2 was that benign training decreased the habit to respond in the same way to analogues of previous negative outcomes. As such, the message is similar to our current report of impairment. Whether by habit or recollection, training seems to impair the use of training-incongruent analogues instead of improving the use of training congruent analogues. The generate/ recognize model does not provide an intuitive fit for our task because a number of words might complete the scenario, and therefore an efficient strategy is simply to wait for the options to be provided on the next screen (permitting the independent contributions of habit and recollection). However, other experimental tasks could be designed to provide estimates of automatic and controlled components that are in line with generate/recognize assumptions or other assumptions about the interaction of automatic and controlled uses of training. ${ }^{2}$

Yet another account of our results relies on the possible use of a strategy for choosing responses when the critical analogue cannot be recalled and participants must therefore guess. In the negative and benign conditions of training, the best guessing strategy might be to respond consistently with their training on same trials and in opposition to training on different trials. This strategy would produce estimates of recollection that are larger when the resolution of critical scenarios is congruent with training and smaller when incongruent with training, compared to estimates in the control condition. In both experiments, this pattern was found for incongruent trials but was nonsignificant for congruent trials. Lacking an explanation for why this guessing strategy would be restricted to incongruent trials, we suggest that its possible use cannot fully account for the differences we found. Nevertheless, evidence of guessing, although difficult to obtain in this paradigm, should be sought in future research. Guessing biases, whether strategic or automatic, are logical loci for effects of prior experience.

Regardless of the model that best characterizes performance in any particular transfer paradigm, we hope that future CBM-I research will continue a procedural analysis of transfer, not only because transfer performance is rarely process pure but also because it is clear that CBM-I affects performance on tests of intentional memory (see Hertel \& Brozovich, 2010). For these and other reasons, investigating the contribution of memory processes to transfer constitutes the sort of direct test of assumptions undergirding CBM that MacLeod et al. (2009) have recently recommended. Not the least of these reasons is the need to know whether emotional benefits of CBM-I are resistant to recollective states of mind, as our findings might suggest.

A final limitation of the experiments we report concerns their inability to address the effects of negative training on recollection of benign outcomes. Only Experiment 1 included a negativetraining condition, and it omitted benign outcomes from the testing procedure. The new tradition of CBM-I experiments began with the idea of simulating interpretations made by anxious individuals. Surely it would be important to know whether pseudoanxious individuals in a negative training condition would have trouble remembering benign outcomes due to proactively interfering interpretations and, moreover, whether such trouble might contribute to the development of anxiety disorders in real life and slow the pace of therapeutic intervention.

\footnotetext{
${ }^{2}$ Proactive interference affected recollection in our experiments; however, Jacoby, Debner, \& Hay (2001) found proactive interference from training on estimates of habit. Their use of PDP sidestepped the instruction to respond differently; all tasks involved recall. We call attention to this difference to illustrate the importance of the particular setting and instructions used in PDP, as well as to suggest possible directions for future research.
}

\section{References}

Beard, C., \& Amir, N. (2008). A multi-session interpretation modification program: Changes in interpretation and social anxiety symptoms. Behaviour Research and Therapy, 46, 1135-1141.

Blanchette, I., \& Richards, A. (2010). The influency of affect on higher level cognition: A review of research on interpretation, judgement, decision making and reasoning. Cognition and Emotion, 24, 561-595.

Ellis, H. C. (1965). The transfer of learning. New York, NY: Macmillan.

Hertel, P. T., \& Brozovich, F. (2010). Cognitive habits and memory distortions in anxiety and depression. Current Directions in Psychological Science, 19, 155-160.

Holmes, E. A., Lang, T. J., \& Shah, D. M. (2009). Developing interpretation bias modification as a "cognitive vaccine" for depressed mood: Imagining positive events makes you feel better than thinking about them verbally. Journal of Abnormal Psychology, 118, 76-88.

Jacoby, L. L. (1991). A process dissociation framework: Separating automatic from intentional uses of memory. Journal of Memory and Language, 30, 513-541.

Jacoby, L. L. (1996). Dissociating automatic and consciously controlled effects of study/test compatibility. Journal of Memory and Language, $35,32-52$.

Jacoby, L. L., Debner, J. A., \& Hay, J. F. (2001). Proactive interference, accessibility bias, and process dissociations: Valid subjective reports of memory. Journal of Experimental Psychology: Learning, Memory, and Cognition, 27, 686-700.

Mackintosh, B., Mathews, A., Yiend, J., Ridgeway, V., \& Cook, E. (2006). Induced biases in emotional interpretation influence stress vulnerability and endure despite changes in context. Behavior Therapy, 37, 209-222.

MacLeod, C., Koster, E. H., \& Fox, E. (2009). Whither cognitive bias modification research? Commentary on the special section articles. Journal of Abnormal Psychology, 118, 89-99.

Mathews, A. (2010). Effects of modifying the interpretation of emotional ambiguity. Manuscript submitted for publication. 
Mathews, A., \& Mackintosh, B. (2000). Induced emotional interpretation bias and anxiety. Journal of Abnormal Psychology, 109, 602-615.

Mathews, A., \& MacLeod, C. (2005). Cognitive vulnerability to emotional disorders. Annual Review of Clinical Psychology, 1, 167-195.

Mitte, K. (2008). Memory bias for threatening information in anxiety and anxiety disorders: A meta-analytic review. Psychological Bulletin, 134, 886-911.

Salemink, E., van den Hout, M., \& Kindt, M. (2007). Trained interpretive bias and anxiety. Behavior Research and Therapy, 45, 329-340.

Spielberger, C. D., Gorsuch, R. L., Lushene, R., Vagg, P. R., \& Jacobs, G. A. (1983). Manual for the State-Trait Anxiety Inventory. Palo Alto, CA: Consulting Psychologists Press.
Wilson, E. J., MacLeod, C., Mathews, A., \& Rutherford, E. M. (2006). The causal role of interpretive bias in anxiety reactivity. Journal of Abnormal Psychology, 115, 103-111.

Zemeckis, R. (Producer/Director), Starkey, S. (Producer), \& Rapke, J. (Producer). (2000). What Lies Beneath [Motion Picture]. United States: DreamWorks.

\section{New Editors Appointed, 2013-2018}

The Publications and Communications Board of the American Psychological Association announces the appointment of 5 new editors for 6-year terms beginning in 2012. As of January 1, 2012, manuscripts should be directed as follows:

- Journal of Experimental Psychology: Learning, Memory, and Cognition (http://www.apa.org/ pubs/journals/xlm/), Robert L. Greene, PhD, Department of Psychology, Case Western Reserve University

- Professional Psychology: Research and Practice (http://www.apa.org/pubs/journals/pro/), Ronald T. Brown, PhD, ABPP, Wayne State University

- Psychology and Aging (http://www.apa.org/pubs/journals/pag), Ulrich Mayr, PhD, Department of Psychology, University of Oregon

- Psychology, Public Policy, and Law (http://www.apa.org/pubs/journals/law/), Michael E. Lamb, PhD, University of Cambridge, United Kingdom

- School Psychology Quarterly (http://www.apa.org/pubs/journals/spq/), Shane R. Jimerson, PhD, University of California, Santa Barbara

Electronic manuscript submission: As of January 1, 2012, manuscripts should be submitted electronically to the new editors via the journal's Manuscript Submission Portal (see the website listed above with each journal title).

Current editors Randi C. Martin, PhD, Michael C. Roberts, PhD, Paul Duberstein, PhD, Ronald Roesch, $\mathrm{PhD}$, and Randy W. Kamphaus, $\mathrm{PhD}$, will receive and consider new manuscripts through December 31, 2011. 